\title{
NONLINEAR DYNAMICS OF PARAMETRICALLY EXCITED CARBON NANOTUBES FOR MASS SENSING APPLICATIONS
}

\author{
S. Souayeh ${ }^{1,2}$, N. Kacem ${ }^{1, *}$, F. Najar ${ }^{2}$ and E. Foltête ${ }^{1}$ \\ ${ }^{1}$ FEMTO-ST Institute, UMR 6174, Applied Mechanics Department, 24 chemin de l'Epitaphe, F-25000 \\ Besançon, France \\ 2 Applied Mechanics and Systems Research Laboratory, Tunisia Polytechnic School BP 743, 2078 La \\ Marsa, Tunisia address \\ *najib.kacem@femto-st.fr
}

Keywords: CNT, Parametric resonance, Nonlinear Dynamics, Mass detection, HBM, ANM.

\begin{abstract}
In this paper, a computational model for large amplitude vibrations of a parametrically excited carbon nanotube (CNT) is developed. The continuous model includes geometric and electrostatic nonlinearities. The Galerkin discretization is used to transform the nonlinear partial differential equation to a finite degrees of freedom system which is numerically solved using the harmonic balance method (HBM) coupled with the asymptotic numerical method (ANM). The influence of higher modes on the nonlinear dynamics of the considered resonator is investigated in order to retain the number of modes which will be used by the HBM+ANM procedure. It is shown that at least two modes are required in order to predict accurately the CNT frequency responses.
\end{abstract}




\section{INTRODUCTION}

Nano electromechanical systems (NEMS) have an important impact in a variety of domains such as the mass detection. Several types of NEMS resonators have been developed like cantilevered sensors, clamped-clamped beams and arrays of beams. Due to their reduced size, NEMS have high frequencies ( $1 \mathrm{GHz}$ ), low power consumption and quality factor in the range of $10^{2}-10^{4}$. These tiny devices permit the investigations of nonlinear properties such as bifurcation topology, bistability and periodic attractors.

The mass spectrometry is the detection of tiny amounts of mass [1] and it is used for highly sensitive tasks. It provides quantitative identification of individual protein species in real time [2]. A Mass spectrometer is composed essentially by three parts: analyte ionization, analyte separation and detection [3]. It allows to achieve femtogram $\left(1 \mathrm{fg}=10^{-15} \mathrm{~g}\right)$, attogram $(1 \mathrm{ag}=$ $\left.10^{-18} \mathrm{~g}\right)$ and zeptogram $\left(1 \mathrm{zg}=10^{-21} \mathrm{~g}\right)$ resolutions. Recently, some devices can reach the mass sensitivity of the range of dalton (1 $D a=1 A M U)$ [4].

When attaching a mass to a resonator, the particle will change the mass response of the sensor. Therefore, the frequency shifts in the fundamental mode of vibration [5]. For an efficient detection of this particle, we should have an accurate method that allows the simultaneous detection of the mass and position. In addition, if we want to reach ultrasensitive sensing, the material composing the sensor plays an important role for precision. Due to the low mass of the nanotube (few attograms), a tiny amount of atoms deposited onto it represents a significant fraction of the total mass. Furthermore, nanotubes are mechanically ultra-rigid permitting the increase of the resonance frequency. Hence, the carbon nanotube (CNT) is the most appropriate device for mass detection applications [6]. Several investigations were done in order to develop a technique allowing the simultaneous position and mass detection. But, such algorithms are very complicated and demand a sophisticated mathematical methods to be solved. The alternative to avoid this problem is to find relations between mass and position of the added particles and the resonant frequencies of the cantilever by measuring the resonant frequencies of the beam without and with added mass for several vibrational modes. In [6], the design of a cantilevered CNT with modeled in the case of the primary resonance.

In this paper, the nonlinear dynamics of a carbon nanotube (CNT) is investigated. To this end, a multiphysics model of a clamped-clamped carbon naotube parametrically excited and including the main sources of nonlinearities is developed. An efficient numerical procedure has been used to investigate the responses of a CNT oscillator for the detection of the mass and the position of an added particle. The main idea is to provide numerical tools for NEMS designers in order to enhance the performances of resonant mass sensors.

Firstly, a design of an electrostatically actuated CNT is proposed and modeled. Then, the system of equations is specifically normalized the electrostatic nonlinearities are expanded up to the fifth order Taylor series in order to take into account all relevant nonlinear terms for NEMS $[7,8,9,10]$. The Galerkin discretization procedure is used in order to transform the multiphysics continuum problem into a finite system of nonlinear ordinary differential equations in time. The reduced-order model is solved numerically using the harmonic balance method coupled with the asymptotic numerical continuation technique. Based on these numerical methods, the frequency responses of the CNT for a specific set of design parameters are derived and investigated in the nonlinear configurations, so that, we can retain the number of modes which gives the most accurate results. Finally, the frequency shifts of the resonance peaks are numerically tracked on the first mode for a particular CNT design and several added masses in different positions along the NEMS length. 


\section{DESIGN AND MODEL}

We consider a carbon nanotube (CNT) resonator depicted in Figure 1. It consists of a single nanobeam with an annular cross section initially straight and clamped at its two ends. It is actuated by two symmetric electrodes providing an electrostatic force $v(\tilde{t})=V_{d c}+V_{a c} \cos (\tilde{\Omega} \tilde{t})$, where $V_{d c}$ is the dc polarization voltage, $V_{a c}$ is the amplitude of the applied ac voltage, $\tilde{t}$ is the time and $\tilde{\Omega}$ is the excitation frequency. The two electrodes are positioned at a distance $d_{1}$ from the fixed end in order to place a piezoelectric or piezoresistive transduction [11] and at a distance $d_{2}$ from the free extremity.

The CNT is modeled as an Euler-Bernoulli beam of length $L$ and with a quality factor $Q$. It has an internal radius $\tilde{R}_{1}$, an external one $\tilde{R}_{2}$.

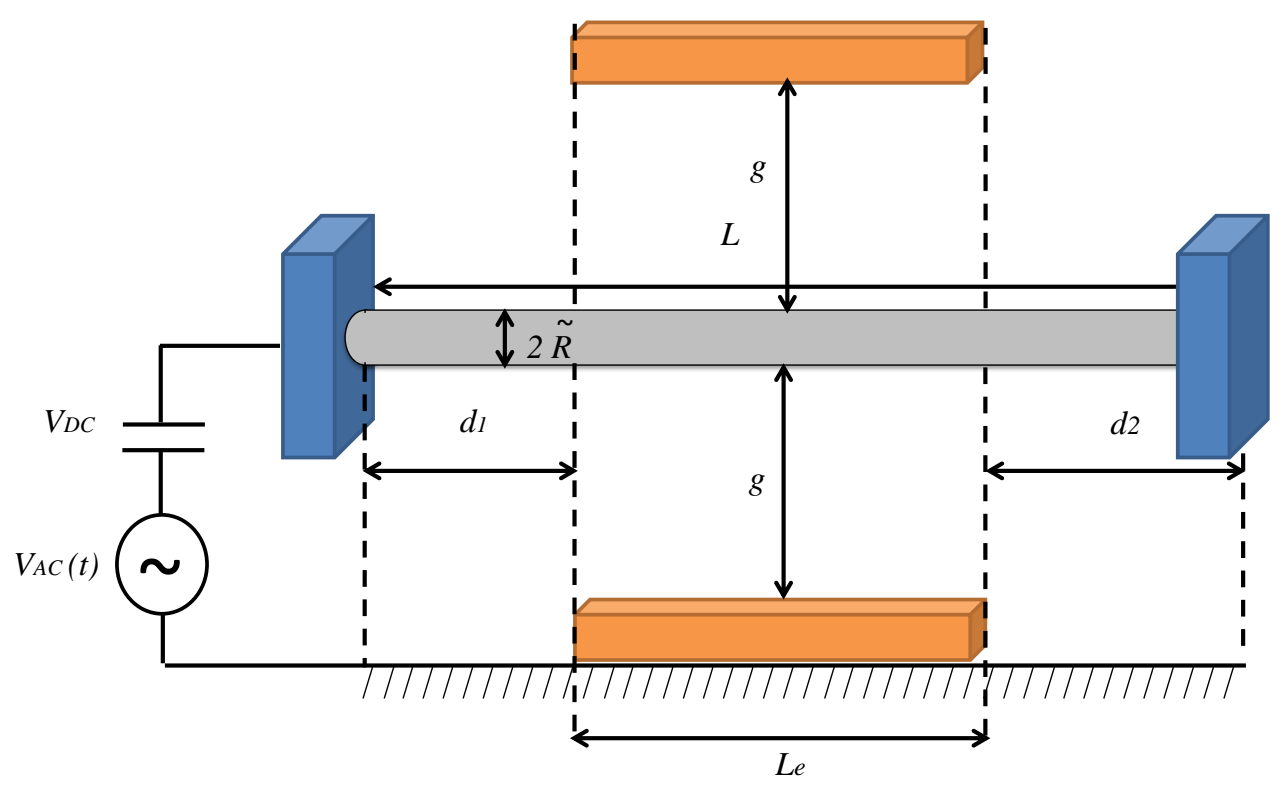

Figure 1: Schematic of a clamped-clamped carbon nanotube electrostatically actuated under parametric resonance.

\section{ANALYTICAL SOLVING}

Let the small and lumped added mass of mass $m_{p}$ and of tiny size fall onto the CNTs surface. The CNT and the added mass constitute a continuum whose bending behavior is governed by the following equation applied to an infinitesimal volume $d \tilde{x}$, with $\delta_{\tilde{x}_{0}}(\tilde{x})$ the Dirac function. The equations of motion of the CNT can be written as [13]:

$$
\begin{aligned}
& E I \partial_{\tilde{x}, \tilde{x}, \tilde{x}, \tilde{x}} \tilde{w}+\rho A \partial_{\tilde{t}, \tilde{t}} \tilde{w}+\tilde{c} \partial_{\tilde{t}} \tilde{w}+\delta_{\tilde{x}_{0}}(\tilde{x}) m_{p} \partial_{\tilde{t}, \tilde{t}} \tilde{w}=\frac{E A}{2 L}\left(\int_{0}^{L}\left(\partial_{\tilde{x}} \tilde{w}\right)^{2} d \tilde{x}\right) \partial_{\tilde{x}, \tilde{x}} \tilde{w}+\mathcal{H}(\tilde{x}) \tilde{F} \\
& \mathcal{H}(\tilde{x})=H\left(\tilde{x}-d_{1}\right)-H\left(\tilde{x}-L+d_{2}\right)
\end{aligned}
$$

where $\partial_{\tilde{x}}$ denotes the partial differentiation with respect to $\tilde{x}$ which is the coordinate along the nanotube length $L, \partial_{\tilde{t}}$ is the partial differentiation with respect to the time $\tilde{t}, \tilde{w}(\tilde{x}, \tilde{t})$ is 
the in-plane bending deflection, $E$ is the effective Young's modulus, $I=\frac{\pi}{4}\left(\tilde{R}_{2}^{4}-\tilde{R}_{1}^{4}\right)$ is the moment of inertia of the circular cross-section, $\rho$ is the density of the nanotube material, $A=\pi\left(\tilde{R}_{2}{ }^{2}-\tilde{R}_{1}{ }^{2}\right)$ is the cross-section area and $\tilde{c}$ is the coefficient of the viscous damping per unit length and $m_{p}$ is the mass of the added particle.

The carbon nanotube is subject to the electrostatic actuation $\mathcal{H}(\tilde{x}) \tilde{F}$, where $\mathcal{H}(\tilde{x})$ includes Heaviside functions $H$ in order to indicate the position of the electrodes with respect to the oscillator, wherein $d_{1}+d_{2}<L$, and $\tilde{F}$ is the electrostatic force expressed as

$$
\tilde{F}=\frac{\pi \epsilon_{0}\left(V_{d c}+V_{a c} \cos (\tilde{\Omega} \tilde{t})\right)^{2}}{\sqrt{(g-\tilde{w})(g-\tilde{w}+2 \tilde{R})}\left(\cosh ^{-1}\left(1+\frac{g-\tilde{w}}{\tilde{R}}\right)\right)^{2}}-\frac{\pi \epsilon_{0}\left(V_{d c}+V_{a c} \cos (\tilde{\Omega} \tilde{t})\right)^{2}}{\sqrt{(g+\tilde{w})(g+\tilde{w}+2 \tilde{R})}\left(\cosh ^{-1}\left(1+\frac{g+\tilde{w}}{\tilde{R}}\right)\right)^{2}}
$$

where $\epsilon_{0}$ is the dielectric constant of the gap medium. The boundary conditions are:

$$
\tilde{w}(0, \tilde{t})=0, \partial_{\tilde{x}} \tilde{w}(0, \tilde{t})=0, \tilde{w}(L, \tilde{t})=0, \partial_{\tilde{x}} \tilde{w}(L, \tilde{t})=0
$$

\subsection{Normalization}

For convenience, Equation (1) is normalized. This normalization is particular for our case because the time constant $\tau$ depends on $V_{d c}$ and ${ }_{k}$ (which we represent later). So, we have $\tau_{k}$ corresponding to each mode. The first step is to introduce the following nondimensional variables into Equation (2):

$$
w=\frac{\tilde{w}}{g}, x=\frac{\tilde{x}}{L}, t=\frac{\tilde{t}}{\tau_{k}}
$$

with $k$ the mode number.

Then, Equation (1) is divided by the coefficient of $\partial_{t, t} w\left(\frac{\rho A g}{\tau^{2}}\right)$ and the electrostatic force is expanded up to the fifth order Taylor series. The Galerkin decomposition method is applied to the obtained equation. To this end, the beam deflection $w(x, t)$ can be written in this form

$$
w(x, t)=\sum_{k=1}^{N_{m}} a_{k}(t) \phi_{k}(x)
$$

where $N_{m}$ is the number of modes retained in the solution, $a_{k}(t)$ is the $k^{\text {th }}$ nondimensional modal coordinate and $\phi_{k}(x)$ is the $k^{t h}$ normalized linear undamped mode shape of a straight beam which is the eigenmode solution of

$$
\frac{d^{4} \phi_{k}(x)}{d x^{4}}=\lambda_{k}^{4} \phi_{k}(x)
$$

where $\lambda_{k}$ is the solution of the transcendental equation

$$
1-\cos \left(\lambda_{k}\right) \cosh \left(\lambda_{k}\right)=0
$$

The modal projection consists in substituting Equation (5) into the equation obtained after the normalization and the division by the coefficient of $\partial_{t, t} w$, multiplying the result by $\phi_{k}(x)$, 
using Equation (6) to eliminate $\frac{d^{4} \phi_{k}(x)}{d x^{4}}$ and integrating the outcome from $x=0$ to 1 . Doing so, we obtain the following equation of motion for the first mode

$$
\begin{aligned}
& a_{i}^{\prime \prime}+c_{i} a_{i}^{\prime}+a_{i}-2 \sum_{j=1}^{N_{m}} \sum_{k=1}^{N_{m}}\left(\int_{0}^{1} \phi_{k} \phi_{j} \phi_{i} d x\right) a_{k} a_{j}^{\prime \prime}+\sum_{j=1}^{N_{m}} \sum_{k=1}^{N_{m}} \sum_{l=1}^{N_{m}}\left(\int_{0}^{1} \phi_{l} \phi_{k} \phi_{j} \phi_{i} d x\right) a_{l} a_{k} a_{j}^{\prime \prime} \\
& -2 \sum_{j=1}^{N_{m}} \sum_{k=1}^{N_{m}}\left(\int_{0}^{1} \phi_{k} \phi_{j} \phi_{i} d x\right) c_{j} a_{k} a_{j}^{\prime}+\sum_{j=1}^{N_{m}} \sum_{k=1}^{N_{m}} \sum_{l=1}^{N_{m}}\left(\int_{0}^{1} \phi_{l} \phi_{k} \phi_{j} \phi_{i} d x\right) c_{j} a_{l} a_{k} a_{j}^{\prime} \\
& -2 \sum_{j=1}^{N_{m}} \sum_{k=1}^{N_{m}}\left(\int_{0}^{1} \phi_{k} \phi_{j} \phi_{i} d x\right) a_{k} a_{j}+\sum_{j=1}^{N_{m}} \sum_{k=1}^{N_{m}} \sum_{l=1}^{N_{m}}\left(\int_{0}^{1} \phi_{l} \phi_{k} \phi_{j} \phi_{i} d x\right) a_{l} a_{k} a_{j} \\
& -\left(N_{0}+\alpha_{1}\left(\sum_{s=1}^{N_{m}} \sum_{p=1}^{N_{m}}\left(\int_{0}^{1} \phi_{s}^{\prime} \phi_{p}^{\prime} d x\right) a_{s} a_{p}\right)\right) \\
& \left(\sum_{j=1}^{N_{m}}\left(\int_{0}^{1} \phi_{j}^{\prime \prime} \phi_{i} d x\right) a_{j}-2 \sum_{j=1}^{N_{m}} \sum_{k=1}^{N_{m}}\left(\int_{0}^{1} \phi_{k} \phi_{j}^{\prime \prime} \phi_{i} d x\right) a_{k} a_{j}+\sum_{j=1}^{N_{m}} \sum_{k=1}^{N_{m}} \sum_{l=1}^{N_{m}}\left(\int_{0}^{1} \phi_{l} \phi_{k} \phi_{j}^{\prime \prime} \phi_{i} d x\right) a_{l} a_{k} a_{j}\right) \\
& =\alpha_{2} \mathcal{H}(x) F_{i}\left(\int_{0}^{1} \phi_{i} d x\right) \\
& \mathcal{H}(x)=H\left(x-\frac{d_{1}}{L}\right)-H\left(x-1+\frac{d_{2}}{L}\right)
\end{aligned}
$$

where $\frac{d_{1}+d_{2}}{L}<1$ and the expressions of the nondimensional parameters introduced in Equation (8) are

$$
\begin{aligned}
& \alpha_{1}=\frac{E g^{2} \tau_{k}^{2}}{2 L^{4} \rho\left(1+\delta_{x_{0}}(x) m\right)}, \alpha_{2}=\frac{\tau_{k}^{2}}{\rho A g^{2}\left(1+\delta_{x_{0}}(x) m\right)} V_{a c} V_{d c}, c=\tilde{c} \frac{\tau_{k}}{\rho A\left(1+\delta_{x_{0}}(x) m\right)}, \\
& \Omega=\tilde{\Omega} \tau_{k}, R_{2}=\frac{\tilde{R}_{2}}{g}
\end{aligned}
$$

with $m=\frac{m_{p}}{\rho A L}$ the mass ratio. The electrostatic force $F_{i}$ is written as

$$
F_{i}=\frac{\pi \epsilon_{0}\left(V_{d c}+V_{a c} \cos (\Omega t)\right)^{2}}{\sqrt{\left(1-a_{i}\right)\left(1-a_{i}+2 R\right)}\left(\cosh ^{-1}\left(1+\frac{1-a_{i}}{R}\right)\right)^{2}}-\frac{\pi \epsilon_{0}\left(V_{d c}+V_{a c} \cos (\Omega t)\right)^{2}}{\sqrt{\left(1+a_{i}\right)\left(1+a_{i}+2 R\right)}\left(\cosh ^{-1}\left(1+\frac{1+a_{i}}{R}\right)\right)^{2}}
$$

Equation (8) can be written in matrix-vector form as

$$
\begin{aligned}
& {\left[M_{0}+M_{1}(a)+M_{2}(a)\right] a^{\prime \prime}+\left[C_{0}+C_{1}(a)+C_{2}(a)\right] a^{\prime}+\left[K_{0}+K_{1}(a)+K_{2}(a)\right] a} \\
& -\left[N_{0}+\alpha_{1} T_{2}(a)\right]\left[K_{T}(a)+K_{T 1}(a)+K_{T 2}(a)\right] a=\alpha_{2} \mathcal{H}(x) F 1
\end{aligned}
$$

where $a(t)=\left[a_{1}(t), a_{2}(t), a_{3}(t), \ldots, a_{N_{m}}(t)\right]^{T}$. The components of matrices $M_{0}, M_{1}, M_{2}$, $C_{0}, C_{1}, C_{2}, K_{0}, K_{1}, K_{2}, K_{T}(a), K_{T 1}(a)$ and $K_{T 2}(a)$ are respectively $M_{0 i j}, M_{1 i j}, M_{2 i j}, C_{0 i j}$, $C_{1 i j}, C_{2 i j}, K_{0 i j}, K_{1 i j}, K_{2 i j}, K_{T i j}, K_{T 1 i j}$ and $K_{T 2 i j}$ : 


$$
\begin{aligned}
& M_{0 i j}=\delta_{i j} \\
& M_{1 i j}=-2 \sum_{j=1}^{N_{m}} \sum_{k=1}^{N_{m}}\left(\int_{0}^{1} \phi_{k} \phi_{j} \phi_{i} d x\right) a_{k} \\
& M_{2 i j}=\sum_{j=1}^{N_{m}} \sum_{k=1}^{N_{m}} \sum_{l=1}^{N_{m}}\left(\int_{0}^{1} \phi_{l} \phi_{k} \phi_{j} \phi_{i} d x\right) a_{l} a_{k} \\
& C_{0 i j}=c_{i} \delta_{i j} \\
& C_{1 i j}=c_{j} M_{1 i j} \\
& C_{2 i j}=c_{j} M_{2 i j} \\
& K_{0 i j}=\delta_{i j} \\
& K_{1 i j}=M_{1 i j} \\
& K_{2 i j}=M_{2 i j} \\
& K_{T i j}=\int_{0}^{1} \phi_{j}^{\prime \prime} \phi_{i} d x \\
& K_{T 1 i j}=-2 \sum_{k=1}^{N_{m}}\left(\int_{0}^{1} \phi_{k} \phi_{j}^{\prime \prime} \phi_{i} d x\right) a_{k} \\
& K_{T 2 i j}=\sum_{k=1}^{N_{m}} \sum_{l=1}^{N_{m}}\left(\int_{0}^{1} \phi_{l} \phi_{k} \phi_{j}^{\prime \prime} \phi_{i} d x\right) a_{l} a_{k}
\end{aligned}
$$

The scalar $T_{2}(a)$ and the entries of $F 1$ are

$$
\begin{aligned}
& T_{2}(a)=\sum_{s=1}^{N_{m}} \sum_{p=1}^{N_{m}}\left(\int_{0}^{1} \phi_{s} \phi_{p} d x\right) a_{s} a_{p} \\
& F 1_{i}=F_{i} \int_{0}^{1} \phi_{i}(x) d x
\end{aligned}
$$

In the following section, the system of equations (11) is solved numerically in order to obtain the frequency responses of the nanotube.

\section{NUMERICAL SIMULATIONS}

In order to compute the periodic solutions of nonlinear differential equations, for nonlinear oscillators, three major steps are followed: the first one consists in transforming the nonlinearities of the original system into quadratic terms. The second one is the decomposition of the quadratic recast equations into truncated Fourier series by means of the harmonic balance method (HBM). The third one is the application of the continuation method (ANM) on the resulting system. At the end, the numerical results are derived. This method [14] is applied on system (11). Thereafter, a detailed description of the quadratic recast and of the combined technique $\mathrm{HBM}+\mathrm{ANM}$ is given.

\subsection{Quadratic recast}

A periodically forced system has this form:

$$
\dot{w}=f(t, w, \Omega)
$$

where $w$ is a vector of unknowns, $f$ is periodic in $t$ and $\Omega$ is a real parameter. To simplify the application of HBM method, the first step is to transform Equation (14) into a new system where the nonlinearities are at most quadratic polynomials as 


$$
m(\dot{Z})=c(t, \Omega)+l(Z)+q(Z, Z)
$$

where $c$ is a constant vector with respect to the unknown $Z, l($.$) is a linear vector with respect$ to the vector entry and $q(.,$.$) is a quadratic vector linear with respect to both entries.$

The following variables are introduced in order to transform Equation (11) into a quadratic system, as described previously.

$$
\begin{aligned}
& v=\dot{a}\left(\text { size } N_{m}\right) \\
& y=\dot{v}\left(\text { size } N_{m}\right) \\
& x=\cos (2 \Omega t)\left(\text { size } N_{m}\right) \\
& M_{t o t}=M_{1}(a)+M_{2}(a)\left(\text { size } N_{m}^{2}\right) \\
& K_{t o t}=K_{T 1}(a)+K_{T 2}(a)\left(\text { size } N_{m}^{2}\right) \\
& S=K_{\text {Ttot }} a\left(\text { size } N_{m}\right) \\
& T=T_{2}(a)(\text { size } 1) \\
& F 1=F F 1_{1}(a)+F F 1_{2}(a)\left(\text { size } N_{m}^{2}\right)
\end{aligned}
$$

System (11) can be rewritten as

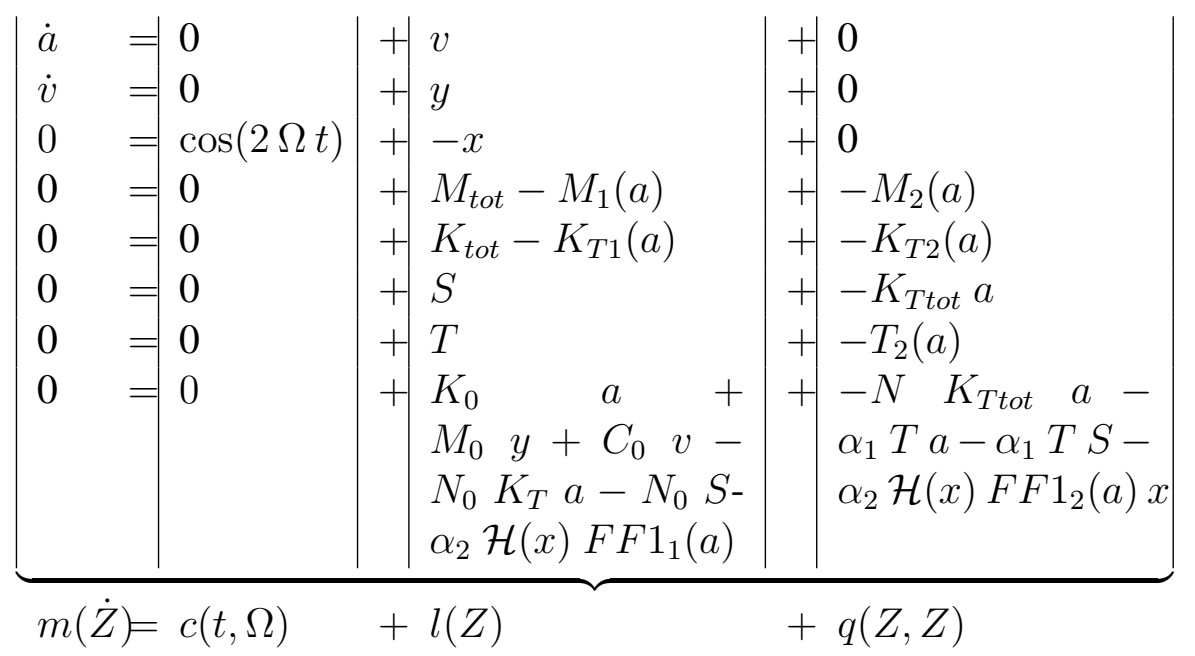

where $Z=\left(a, v, y, M_{t o t}, S, T, F 1\right)^{T}$ is the unknown vector of size $N_{e q}=4 N_{m}+3 N_{m}^{2}+$ $1, c$ is a constant vector with respect to $Z, l($.$) and m($.$) are linear vectors valued operators$ with respect to $Z$, and $q(.,$.$) is a quadratic vector. In our case, for two modes, N_{e q}=21$ corresponding to the number of equations of system (17).

\subsection{The harmonic balance method (HBM)}

The harmonic balance method is now applied to the system of Equations (17). The unkown vector $Z$ is decomposed into Fourier series with $\mathrm{H}$ harmonics

$$
Z(t)=Z_{0}+\sum_{k=1}^{H} Z_{c, k} \cos (k \omega t)+\sum_{k=1}^{H} Z_{s, k} \sin (k \omega t)
$$

Then, column vector $U$, with size $(2 H+1) \times N e q$, where $N e q$ is the number of equations in Equation (17), collects the components of the Fourier series as 


$$
U=\left[Z_{0}^{t}+Z_{c, 1}^{t}+Z_{s, 1}^{t}+Z_{c, 2}^{t}+\ldots+Z_{c, H}^{t}+Z_{s, H}^{t}\right]^{t}
$$

Substituting Equation (19) into Equation (17), collecting the terms of the same harmonic index and neglecting the higher order harmonics, we obtain this set of equations

$$
\omega M(U)=C+L(U)+Q(U, U)
$$

It contains $(2 H+1) \times N e q$ equations for the $(2 H+1) \times N e q$ unknowns $U$. Operators $M($.$) ,$ $C, L($.$) , and Q(.,$.$) depend only on the operators m(), c,. l($.$) and q(.,$.$) of Equation (17) and$ on the number of harmonics $H$.

\subsection{The continuation procedure}

From Equation (20), an algebraic system is obtained

$$
R(U)=0
$$

where $R \in R^{N+1}$ and $U=\left[U^{t}, \Omega, \omega\right] \in R^{N+1}$. The Asymptotic numerical method (ANM), which is based on the quadratic recasting, is applied to Equation (20) to obtain

$$
R(U)=L 0+L(U)+Q(U, U)
$$

where $L 0, L($.$) and Q(.,$.$) are respectively constant, linear and bilinear vectors. Then, the$ solutions are obtained by considering the branches of solution as power series. Indeed, if $U_{0}$ is a point solution, the branch passing by $U_{0}$ is a power series expansion of the path parameter $a=$ $\left(U-U_{0}\right)^{t} U_{1}$, where $U_{1}$ is the tangent vector at $U_{0}$ and $U(a)=U_{0}+a U_{1}+a^{2} U_{2}+a^{3} U_{3}+\ldots+a^{n} U_{n}$. This serie is replaced in Equation (21) where the powers of $a$ are equated to zero providing a set of linear systems.

\section{RESULTS AND DISSCUSSIONS}

Due to the symmetry of the problem, the odd modes are retained. Due to the kind of the dominant nonlinearities, the even harmonics have no influence. In addition and after verifications, the first harmonic gives the most important informations. So, in our case, we have plotted the amplitudes $W_{\max -i}, i \in\{1,3\}$, of the first, the third and the fifth modes normalized by the gap $g$ at $x=L$ and corresponding to the first harmonic.

The frequency responses of the first mode of the carbon nanotube's configuration of table 1 , without added mass, are represented in Figure 2 when using one or two modes, in order to show the impact of each mode on the frequency responses and to retain the most appropriate number of used modes.

The amplitudes of the two frequency responses are remarkably different. Indeed, for a single mode the amplitude is equal to $0.27 \%$ of the gap, however, with two modes the amplitude is equal to $0.17 \%$ of the gap. Hence, we have to use at least two modes in order to obtain the most accurate results.

Now, we track the frequency response of the first mode when adding a mass at different positions on the CNT. The two frequencies have a hardening behavior when using a single or 
Table 1: Design parameters of investigated carbon nanotube resonator.

\begin{tabular}{clllllll}
\hline Resonator & $L(\mu m)$ & $R_{1}(\mathrm{~nm})$ & $R_{2}(\mathrm{~nm})$ & $g(\mathrm{~nm})$ & $V_{a c}(V)$ & $V_{d c}(V)$ & $Q$ \\
\hline 1 & 40 & 350 & 500 & 200 & 3 & 15 & 900 \\
\hline
\end{tabular}

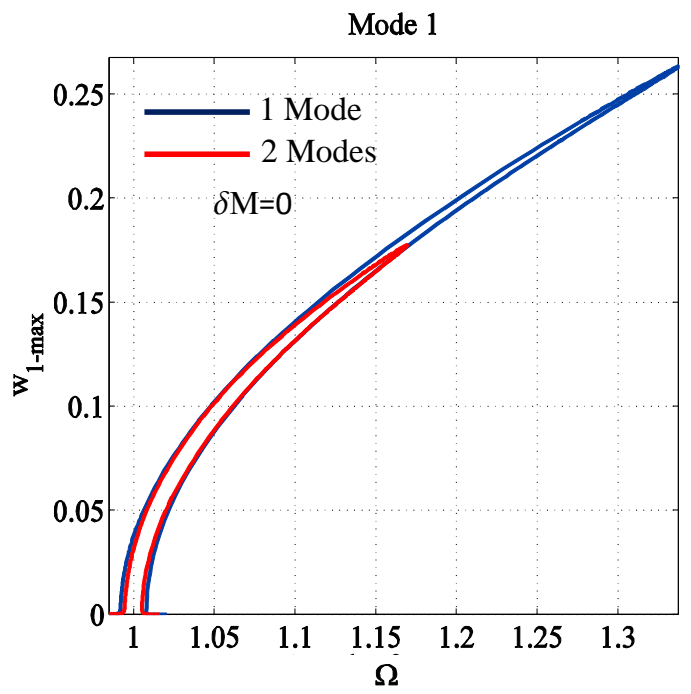

Figure 2: Frequency responses of the first mode when using one and two modes without added mass.

several modes. For our case, the positions can be limited between a clamped end and the middle of the clamped-clamped nanotube because the two halves of the resonator are symmetric as shown in Figure 1. In Figure 3, we represent the frequency responses of the first mode of the CNT (table 1) when adding a mass $m=0.02$ at two different positions $\left(\delta_{x}=0.5\right.$ and $\left.\delta_{x}=0.1\right)$ for several modes.
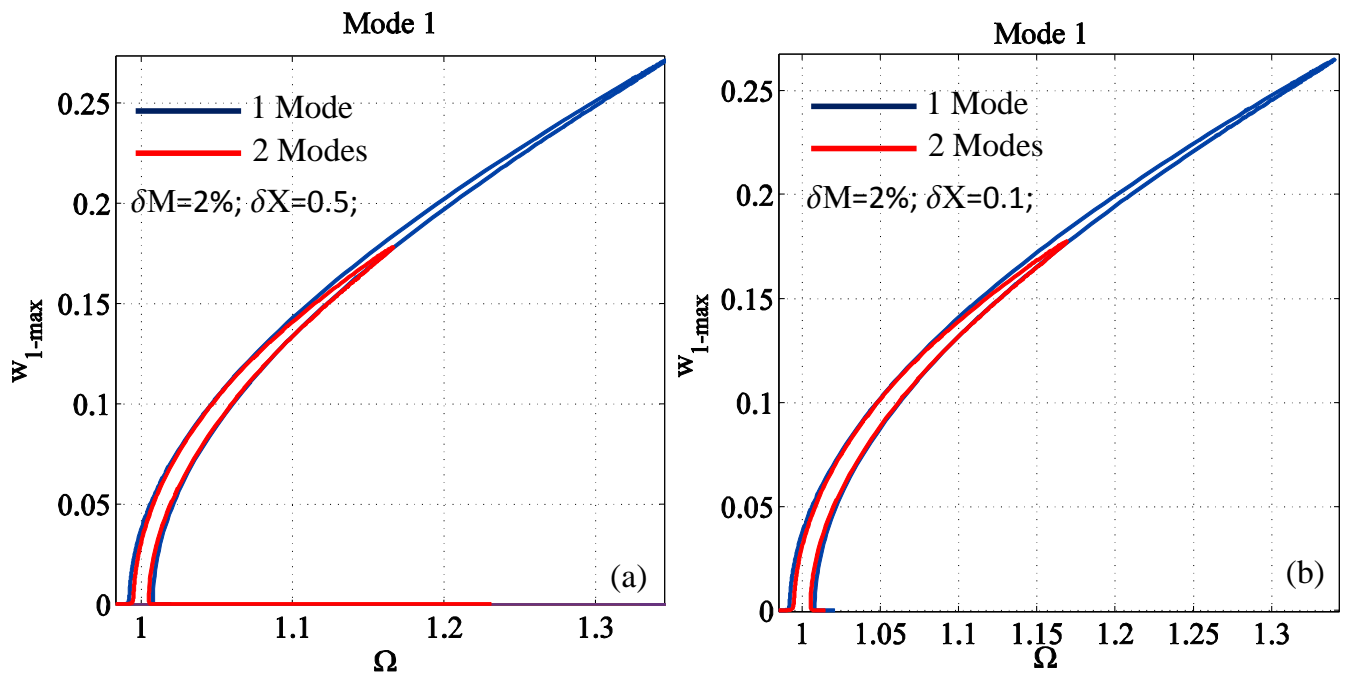

Figure 3: Frequency responses of the first mode when using one and two modes with (a) $\delta_{M}=2 \%, \delta_{x}=0.5$ and (b) $\delta_{M}=2 \%, \delta_{x}=0.1$.

The amplitudes of the two curves increase for the two cases (Figures 3 (a) and (b)). Indeed, when a particle is placed at a specific position along the neutral axis of the nanotube, the natural 
frequencies decrease producing an increase on the amplitudes of the frequency responses.

\section{CONCLUSION}

In this paper, we modeled the nonlinear dynamics of a clamped-clamped carbon nanotube excited under parametric resonance and including geometric and electrostatic nonlinearities. Using the Galerkin discretization method, the Euler-Bernoulli partial differential equation describing the nonlinear motion of the resonator is transformed into a system of coupled nonlinear ordinary differential equations.

Firstly, we developed a numerical multimodal approach based on the harmonic balance method (HBM) and the asymptotic numerical method (ANM). It was demonstrated, for several design parameters, that the use of a single mode is not sufficient to capture the main nonlinear phenomena of the considered device and for mass detection, at least two modes are required.

\section{REFERENCES}

[1] J. Verd, A. Uranga, G. Abadal, J. Teva, F. Torres, M. F. Perez, J. Fraxedas, J. Esteve, N. Barniol, Monolithic mass sensor fabricated using a conventional technology with attogram resolution in air conditions, Applied Physics Letters, 91, 013501, 2007.

[2] M. S. Hanay, S. Kelber, A. K. Naik, D. Chi, S. Hentz, E. C. Bullard, E. Colinet, L. Duraffourg, M. L. Roukes, Single-protein nanomechanical mass spectrometry in real time, Nature nanotechnology, 2012.

[3] A. K. Naik, M. S. Hanay, W. K. Hiebert, X. L. Feng, M. L. Roukes, Towards singlemolecule nanomechanical mass spectrometry, Nature nanotechnology, 4, 445-450, 2009.

[4] H. Chiu, P. Hung, H. W. C.Postma, M. Bockrath, Atomic-scale mass sensing using carbon nanotube resonators, Nano Letters, 8, 533-537, 2008.

[5] K. L. Ekinci, Y. T. Yang, M. L. Roukes, Ultimate limits to inertial mass sensing based upon nanoelectromechanical systems, Journal of Applied Physics, 95, 2682-2689, 2004.

[6] S. Souayeh, N. Kacem, Computational models for large amplitude nonlinear vibrations of electrostatically actuated carbon nanotube-based mass sensors, Sensors and Actuators A: Physical, 208, 10-20, 2014.

[7] N. Kacem, S. Hentz, D. Pinto, B. Reig, V. Nguyen, Nonlinear dynamics of nanomechanical beam resonators: improving the performance of NEMS-based sensors. Nanotechnology, 20(27), 275501, 2009.

[8] N. Kacem, S. Hentz, Bifurcation topology tuning of a mixed behavior in nonlinear micromechanical resonators. Applied Physics Letters, 95(18), 183104, 2009.

[9] N. Kacem, J. Arcamone, F. Perez-Murano, S. Hentz, Dynamic range enhancement of nonlinear nanomechanical resonant cantilevers for high sensitive NEMS gas/mass sensors applications. Journal of Micromechanics and Microengineering, 20(4), 045023, 2010.

[10] N. Kacem, S. Baguet, R. Dufour, S. Hentz, Stability control of nonlinear micromechanical resonators under simultaneous primary and superharmonic resonances. Applied Physics Letters, 98(19), 193507, 2011. 
[11] E. Mile, G. Jourdan, I. Bargatin, C. Marcoux, P. Andreucci, S. Hentz, C. Kharrat, E. Colinet, L. Duraffourg, In-plane nanoelectromechanical resonators based on silicon nanowire piezoresistive detection, Nanotechnology, 21, 165504, 2010.

[12] A. Nayfeh, M. Younis, E. Abdel Rahman, Dynamic pull-in phenomenon in MEMS resonators, Nonlinear Dynamics, 48, 153-163, 2007.

[13] H. M. Ouakad, M.I. Younis, Nonlinear dynamics of electrically actuated carbonnanotube resonators. ASME Journal of Computational and Nonlinear Dynamics, 5, 1-13, 2010.

[14] B. Cochelin, C. Vergez, A high order purely frequency-based harmonic balance formulation for continuation of periodic solutions, Journal of sound and vibration 324, 243-262, 2009. 